\title{
Intrinsic Charm in B-Meson Decays
}

SLAC-PUB-9490

$\mathrm{UK} / \mathrm{TP}$ 02-14

\author{
S. Gardner a b * \\ a Stanford Linear Accelerator Center, Stanford University, Stanford, CA 94309 USA \\ b Department of Physics \& Astronomy, University of Kentucky, Lexington, KY 40506-0055 USA
}

Light cone hadron wave functions support Fock states of arbitrarily high particle number: their heavy quark content arises naturally through QCD interactions. We discuss what role $c \bar{c}$ pairs, intrinsic to a hadron's structure, can play in B-meson decays. The effects can be prominent in hadronic decays for which the tree-level contributions are Cabibbo-suppressed, as in $B \rightarrow \pi K$ decay, and they mimic "charming penguin" contributions.

\section{INTRODUCTION}

It is usually assumed in the analysis of $B$-meson decays that only the valence quarks of the initial and final-state hadrons participate in the weak transition. Any non-valence gluon or sea quarks present in the initial or final state wave functions appear only as spectators. However, the wave functions of a bound state in a relativistic quantum field theory such as QCD necessarily contain Fock states of arbitrarily high particle number. This takes on new significance in light of the hierarchical structure of the CKM matrix - the weak transition $b \rightarrow s c \bar{c}$ is doubly Cabibbo enhanced with respect to a $b \rightarrow s u \bar{u}$ transition. The small probability of realizing a Fock state containing a $c \bar{c}$ pair is offset by the comparatively large CKM matrix elements associated with the $b \rightarrow s c \bar{c}$ transition, promoting its phenomenological impact. In this talk, I discuss how the presence of intrinsic charm in the hadrons' light-cone wave functions, even at a few percent level, can impact B-meson decays. My remarks are based on work done in collaboration with Stan Brodsky [1].

To begin, we define intrinsic charm (IC) and review the evidence for its presence in the light hadrons, arguing that the magnitude of IC could be larger in the B-meson. We proceed to consider

\footnotetext{
*Invited talk at the 5th International Conference on $\mathrm{Hy}$ perons, Charm and Beauty Hadrons (BEACH 2002), University of British Columbia, Vancouver, June 25-29, 2002.
}

the disparate roles for IC in B physics, reviewing how it can mediate certain, rare decays, how it can enter the semi-leptonic branching ratio cum "charm counting" puzzle, and finally how it can act as a source of hadronic pollution, impacting the extraction of $\gamma$ in $B \rightarrow \pi K$ decays.

\section{INTRINSIC CHARM?}

The wave functions of a relativistic bound state contain Fock states of arbitrarily high particle number. For example,

$$
\begin{gathered}
\left|B^{-}\right\rangle=\psi_{b \bar{u}}|b \bar{u}\rangle+\psi_{b \bar{u} g}|b \bar{u} g\rangle+\psi_{b \bar{u} d \bar{d}}|b \bar{u} d \bar{d}\rangle \\
+\psi_{b \bar{u} c \bar{c}}|b \bar{u} c \bar{c}\rangle+\cdots
\end{gathered}
$$

The Fock state decomposition is usually performed at equal light-cone time using light-cone quantization in light-cone gauge $A^{+}=0$ [2, 3 . The non-valence partons of the higher Fock states arise from QCD interactions. The partons of a Fock component are entangled through multiple gluon interactions, as illustrated in Fig. 1; this makes them intrinsic to the hadron's structure. The intrinsic, heavy quarks are part of the hadron's non-perturbative bound-state [4].

In contrast, a perturbative correction to the weak transition matrix element can yield a $c \bar{c}$ pair through gluon splitting; the quark pair is generally not multiply connected to the partons of the bound state and thus is extrinsic to the hadron's structure, as illustrated in Fig. 2. 


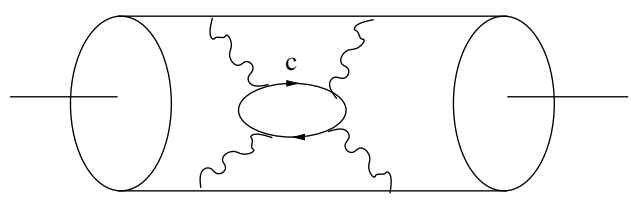

Figure 1. An intrinsic charm contribution to a meson's self-energy.

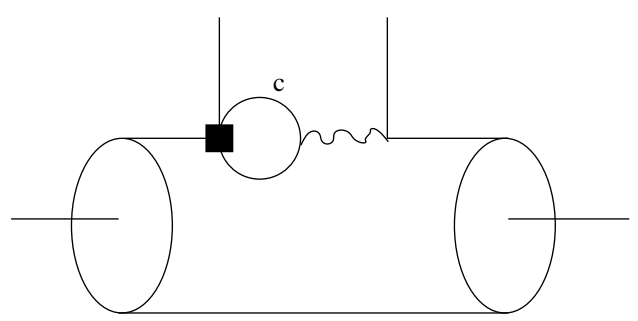

Figure 2. Extrinsic charm as a radiative correction to the effective Hamiltonian, whose action is denoted by the square box, for $B \rightarrow h_{1} h_{2}$ decay.

Generally, "intrinsic" contributions in B-meson decay are of higher twist than the leading contribution, whereas "extrinsic" contributions are of higher order in $\alpha_{s}$ : they may not be crisply separable.

\subsection{Evidence for intrinsic charm}

Deeply inelastic scattering (DIS) measurements at large momentum transfer, $Q^{2} \gg$ $1(\mathrm{GeV} / \mathrm{c})^{2}$, reveal the proton to have a rich sea structure. Our interest is in observables which can distinguish the perturbative evolution of the proton's structure functions from "intrinsic" effects. For example, the proton's flavor structure permits it to fluctuate to $K^{+} \Lambda$, making the $s$ and $\bar{s}$ parton distribution functions unequal [5]. This effect has recently been observed [6], so that intrinsic effects are appreciable, but what of intrinsic charm? The intrinsic heavy-quark fluctuations in hadrons can be analyzed using the operatorproduct expansion: Franz et al. determine that the momentum fraction carried by the heavy $Q \bar{Q}$ pair scales as $1 / m_{Q}^{2}$ ], where we assume that the associated hadronic matrix element is characterized by $k_{\perp}^{2}$, where $k_{\perp}$ is a typical momentum scale in the hadron light-cone wave function. In contrast, in Abelian theories, the contribution of an intrinsic, heavy lepton pair to the bound state's structure first appears in $\mathcal{O}\left(1 / m_{L}^{4}\right)$. Franz et al. estimate that the intrinsic charm probability in the proton is $\lesssim 1 \%$ [7]. Analyses of DIS data are consistent with this prediction [8]. Phenomenologically, the presence of IC at the $\lesssim 1 \%$ level is appealing. IC naturally explains the " $\rho \pi$ puzzle" in $J / \psi\left(\psi^{\prime}\right)$ decays [9], and an explicit IC component is needed to describe the $x_{F} \rightarrow 1$ charm production and polarization data in $\pi N$ reactions 10 .

\subsection{Intrinsic charm in the B-meson}

The existence of intrinsic charm (IC) in the proton implies that IC exists in other hadrons as well. In order to translate an estimate of the IC probability in the proton to that of the IC of a $B$ meson, we are faced with two conflicting effects. The typical internal transverse momentum $k_{\perp}$ is larger in the B-meson, evidently favoring a larger IC probability in the B meson; on the other hand, the proton's additional valence quark generates a larger combinatoric number of IC diagrams, favoring a larger IC probability in the proton. The magnitude of $k_{\perp}$ is significantly larger in the Bmeson; $\lambda_{1}$, the kinetic energy of the $\mathrm{b}$ quark in the B-meson, is $\sqrt{\left|\lambda_{1}\right|} \sim 0.4 \mathrm{GeV} 11$. Minimizing the light-cone energy of the partons in the IC Fock component of the free light-cone Hamiltonian lead Chang and Hou to estimate that the momentum fraction carried by charm is smaller in the B-meson, namely $\left\langle x_{c}\right\rangle \approx 0.22$, than in the proton, for which $\left\langle x_{c}\right\rangle \approx 0.28$ [12]. Thus the IC probability must be higher in the B-meson to make the charmed quarks carry the same, fixed fraction of the hadron's momentum. These estimates suggest that the IC probability in the Bmeson could be as large as a few percent, and the presence of IC in the $\Lambda_{b}$ baryon could be larger still.

\section{ROLES FOR INTRINSIC CHARM IN B-DECAY}

Intrinsic charm can play a variety of roles in Bmeson decays: it can act as a mediator of certain, rare decays; it may give new insight on old (resolved?) puzzles; and it may impact the extrac- 
tion of CKM information from decays to strange, charmless final states. We consider each of these roles in turn.

The presence of intrinsic charm quarks in the $B$ wave function provides new mechanisms for $B$ decays. For example, the production of final states with three charmed quarks, $b \rightarrow c \bar{c} c X$, such as $\bar{B} \rightarrow J / \psi D \pi$ and $\bar{B} \rightarrow J / \psi D^{(*)}$ [12], are difficult to realize in a valence model, as the $c \bar{c}$ pair can only be realized through OZI-violating processes. They occur naturally, however, when the $b$ quark of the intrinsic charm Fock state $|b \bar{u} c \bar{c}\rangle$ decays via $b \rightarrow c \bar{u} d$ - the intrinsic $c \bar{c}$ component of the $\mathrm{B}-\mathrm{meson}$ is materialized in the final state. Chang and Hou suggest that the slight excess in the inclusive $B \rightarrow J / \psi X$ yield at low $J / \psi$ momentum, observed by CLEO [13], hints to the presence of $\bar{B} \rightarrow J / \psi D^{(*)}$ [12], though such an effect could also be generated by $\bar{B} \rightarrow J / \psi \Lambda \bar{n}$ decay [14]. Intrinsic charm in the $\mathrm{B}$ meson can also mediate $B \rightarrow J / \psi \gamma$ and $B \rightarrow J / \psi e^{-} \bar{\nu}_{e}$ decays. Numerical estimates have been made for $\bar{B}^{0} \rightarrow J / \psi X e^{-} \bar{\nu}_{e}$ and $\bar{B}^{0} \rightarrow D \bar{D} X e^{-} \bar{\nu}_{e}$ decay; the branching ratios are markedly larger when IC in the B-meson is included, though they remain small, note, e.g., $\mathcal{B}\left(B^{-} \rightarrow J / \psi e^{-} \bar{\nu}_{e} X\right) \approx 4 \cdot 10^{-7} 115$.

Intrinsic charm could well prove helpful in resolving long-standing puzzles in B-physics. For example, the observed lifetime ratio of the $B$ and $\Lambda_{b}$ hadrons, $\tau\left(\Lambda_{b}\right) /\left.\tau(B)\right|_{\text {expt }}=0.797 \pm 0.053$ [16], differs significantly from unity, the result predicted in the heavy-quark limit. Spectator effects, i.e., the manner in which the decaying $b$ quark interacts with its hadronic environment, are evidently crucial to explaining the discrepancy [17]; spectator interactions involving intrinsic charm ought differ in the $B$ and $\Lambda_{b}$ and could play a role.

Moreover, the semileptonic branching fraction in inclusive B-meson decay, $\mathrm{B}_{\mathrm{sl}}$, is a bit low with respect to SM predictions; however, the "natural" resolution of this puzzle - an increased $b \rightarrow s c \bar{c}$ rate - is untenable, as the observed number of charm (and anti-charm) quarks per Bmeson decay, $n_{c}$, is consistent with SM expectations [18. That is, $\mathrm{B}_{\mathrm{sl}}$ would decrease were the hadronic width of the B-meson to increase, but the hadronic width is tied to $n_{c}$, the average yield

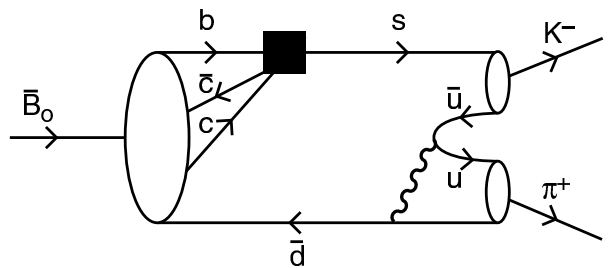

7-2001
8610A1

Figure 3. Intrinsic charm in the B-meson can mediate the decay to a strange, charmless final state via the weak transition $b \rightarrow s c \bar{c}$.

of $c$ and $\bar{c}$ per B-meson decay. IC in the B-meson can increase the charmless decay rate, as in the exclusive process shown in Fig. 3, thus reducing the semileptonic branching ratio. IC thus acts to loosen the correlation between the hadronic width, particularly the $b \rightarrow s c \bar{c}$ rate, and $n_{c}$. Earlier work ascribed a possible role to IC in resolving the $B_{\mathrm{sl}}$ cum $n_{c}$ puzzle [19], yet only IC in the light hadrons was considered. The role played by IC in the B meson in realizing strange, charmless final states may be of greater importance.

Finally, IC could be important to understanding the empirical $B \rightarrow \eta^{\prime} K, \eta^{\prime} X$ branching ratios, which are large with respect to SM estimates. Previously, a valence $c \bar{c}$ component in the $\eta^{\prime}$ had been invoked to resolve the disparity 20], but the decay constant $f_{\eta^{\prime}}^{(c)}$, namely $\left\langle 0\left|\bar{c} \gamma_{\mu} \gamma_{5} c\right| \eta^{\prime}(p)\right\rangle \equiv i f_{\eta^{\prime}}^{(c)} p_{\mu}$, is too small [7]; efforts to reconcile the observed rate with SM predictions continue. Although other mechanisms could well be at work 21], the factorization approximation does not capture the physics of IC. IC is produced in a higher Fock component of a hadron's light-cone wave function; it is naturally in a color octet state [22], so that the dynamical role it plays in mediating B-meson decay is intrinsically non-factorizable in nature.

\subsection{Intrinsic charm in $B \rightarrow \pi K$ decays}

We now turn to the role of intrinsic charm in $B \rightarrow \pi K$ decay. These decays are penguin dominated as $b \rightarrow s u \bar{u}$ decay is $\mathcal{O}\left(\lambda^{2}\right)$ suppressed. The $|\Delta B|=1$ effective Hamiltonian for $b \rightarrow s q \bar{q}$ decay 
is

$$
\begin{gathered}
\mathcal{H}_{\mathrm{eff}}=\frac{G_{F}}{\sqrt{2}}\left\{\sum_{p=u, c}\left[V_{p b} V_{p s}^{*}\left(C_{1} O_{1}^{p}+C_{2} O_{2}^{p}\right)\right]\right. \\
\left.-V_{t b} V_{t s}^{*} \sum_{j} C_{j} O_{j}\right\}
\end{gathered}
$$

where $C_{2} \sim \mathcal{O}(1)$. Sums of the products of $C_{i}(\mu)$ and the matrix elements of $O_{i}(\mu)$ are renormalization scale and scheme invariant, so that each such entity is a parameter: the set constitutes a parametrization [23]. For $B \rightarrow \pi K$ decay, in valence approximation, the decay amplitude $\mathcal{A}\left(B^{0} \rightarrow K^{+} \pi^{-}\right)$, e.g., can be written 23

$$
\begin{gathered}
\mathcal{A}\left(B^{0} \rightarrow K^{+} \pi^{-}\right)=V_{u s} V_{u b}^{*}\left[E_{1}-P_{1}^{\mathrm{GIM}}\right] \\
-V_{t s} V_{t b}^{*} P_{1} .
\end{gathered}
$$

The parameter $E_{1}$ contains $W^{+}$emission topologies, $P_{1}$ contains penguin topologies, and $P_{1}^{\mathrm{GIM}}$ contains penguin contributions which vanish in the $m_{c}=m_{u}$ limit. Beyond valence approximation, the form of the parametrization does not change, but additional contributions arise. That is, from IC, we have the additive contribution $V_{c s} V_{c b}^{*} A_{1}^{\mathrm{IC}}$, where

$A_{1}^{\mathrm{IC}}=C_{1}\left\langle O_{1}^{c}\right\rangle^{\mathrm{IC}}+C_{2}\left\langle O_{2}^{c}\right\rangle^{\mathrm{IC}}$,

to $\mathcal{A}\left(B^{0} \rightarrow K^{+} \pi^{-}\right)$- a contribution of this ilk is illustrated in Fig. 3. The IC contribution is Cabibbo-enhanced and contains an $\mathcal{O}(1)$ Wilson coefficient, just as "charming penguins" do [24,25], so that charming penguins need not be penguins at all. Indeed, the parameter $A_{1}^{\mathrm{IC}}$ can be absorbed into $P_{1}$ and $P_{1}^{\mathrm{GIM}}$. If the contributions are driven by non-perturbative physics, as Ciuchini et al. argue [24], the contributions are probably indistinguishable. We now proceed to the evaluation of the parameters we have introduced.

In the usual perturbative QCD treatment of exclusive processes, the amplitude for a particular exclusive process is formed by the convolution of the nonperturbative distribution amplitudes, $\phi_{H}(x, Q)$, with the hard scattering amplitude, $T_{H}$, computed from the scattering of onshell, collinear quarks [26]27. For $B \rightarrow M_{1} M_{2}$ decay, we have

$$
\begin{gathered}
\mathcal{M}\left(B \rightarrow M_{1} M_{2}\right)=\int_{0}^{1} d z \int_{0}^{1} d y \int_{0}^{1} d x \phi_{B}(x, Q) \\
T_{H}(x, y, z) \phi_{M_{1}}(y, Q) \phi_{M_{2}}(z, Q),
\end{gathered}
$$

where, e.g., $\phi_{M_{2}}(z, Q)=\int_{0}^{Q} d^{2} k_{\perp} \phi\left(x, k_{\perp}, \lambda_{i}\right)$. This formula is suitable if the distribution amplitudes vanish sufficiently rapidly at the endpoints, and if $\alpha_{s}(\mu)$ is sufficiently small for a perturbative treatment to be germane; however, these criteria do not appear to be satisfied in $B \rightarrow \pi l \nu_{l}$ decay [28,29]. Equation (5) itself emerges from an expansion of $T_{H}$ in powers of $k_{\perp}^{2} / Q^{2}$; one solution [30] is to reorganize the contributions in $k_{\perp}$, so that the contributions to the hard scattering in the transverse configuration space ( $b$, conjugate to $k_{\perp}$ ) are no longer of point-like size. The $b$ dependence of the reorganized distribution amplitudes, the so-called "Sudakov exponent," suppresses the large $b$ region, so that the resulting integrals are convergent and $\alpha_{s}(\mu)$ is more or less consistently small. These ideas have been extended to B-meson decays by $\mathrm{Li}$ and collaborators: the $B \rightarrow \pi$ form factor is regarded as perturbatively calculable, once proper resummation techniques are applied 31].

In the approach of Beneke et al. [32], however, the $B \rightarrow \pi$ form factor is treated as nonperturbative input. One consequence is that the perturbative corrections in the two schemes are organized differently: in Ref. [32], hard-scattering contributions in $\mathcal{O}\left(\alpha_{s}\right)$ are retained which would be regarded as non-leading order in the approach of Ref. [31]. However, infrared enhancements also plague the treatment of annihilation contributions in this approach [32]; thus the treatment of Ref. [31] is more systematic in that the contributions of all the decay topologies are regulated in the same way. The internal consistency of the $\mathrm{Li}$ et al. framework has, however, been criticized [1, 33]. We shall nevertheless adopt it in the discussion to follow.

Adopting the notation and conventions of Ref. 34, the parameters of Eq. (3) can be mapped to

$$
\begin{gathered}
E_{1}=-f_{K} F_{e}-M_{e} ; \quad P_{1}^{\mathrm{GIM}}=0, \\
P_{1}=-f_{K} F_{e}^{P}-M_{e}^{P}-f_{B} F_{a}^{P}-M_{a}^{P},
\end{gathered}
$$

where "factorizable" and "non-factorizable" contributions are denoted by $F$ and $M$, respectively. The subscripts $e$ and $a$ refers to emission and annihilation topologies, respectively, and the $P$ superscript reflects the presence of penguin opera- 
tors in the hard-scattering amplitude. The $F$ and $M$ form factors are calculated in leading order in $\alpha_{s}$; in this order, the "extrinsic charm" contribution of Fig. 2 does not occur. The results of including the IC contribution, as per Ref. [1],

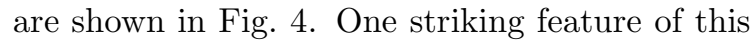
approach is the large direct CP asymmetry predicted in $B^{0} \rightarrow K^{+} \pi^{-}$decay [34,36], in distinction to the predictions of Ref. [32]. Experiment does not currently favor either scenario [37]. Note that IC can act to either enhance or decrease the $\mathrm{CP}$ asymmetry; IC, or as yet uncomputed NLO effects, could mute the distinction between the two approaches. Moreover, the IC contribution we estimate, $\left|A_{1}^{\mathrm{IC}}\right| /\left|P_{1}\right| \sim \mathcal{O}(10) \%$, is a non-trivial fraction of the penguin parameter $P_{1}$; its presence casts doubt on the ability to calculate the effective value of $P_{1}$. Moreover, a factorization assumption for the effective penguin contribution in $B^{+} \rightarrow K_{0} \pi^{+}$decay, needed for an $\mathrm{SU}(3)_{f}$-based analysis of the time-dependent asymmetries in $B \rightarrow \pi^{+} \pi^{-}$for $\sin (2 \alpha)$ [38], may not be warranted.

\section{SUMMARY}

The role of non-valence components in the hadrons' light-cone wavefunctions, coupled with the hierarchical structure of the CKM matrix, offers new perspective on $\mathrm{B}$ decays. The effects can be striking in exclusive decays with Cabibbosuppressed tree contributions, thus our focus on intrinsic charm in $B \rightarrow \pi K$ decay. Intrinsic charm effects are quantitatively elusive, but can be expected to play a non-trivial role in decays to strange, charmless final states. They may prove inseparable from charming penguin contributions. Such effects can modify the effective value of $P_{1}$, the penguin contribution, from its expected value, to confound the determination of $\gamma$. The observation of the rare decay $B \rightarrow J / \psi D X$ decay, or any of its brethren, would be clarifying.

ACKNOWLEDGEMENTS It is a pleasure to acknowledge Stan Brodsky for an engaging and fruitful collaboration, as well as the SLAC Theory Group for its hospitality. I thank the conference organizers for the invitation to speak and

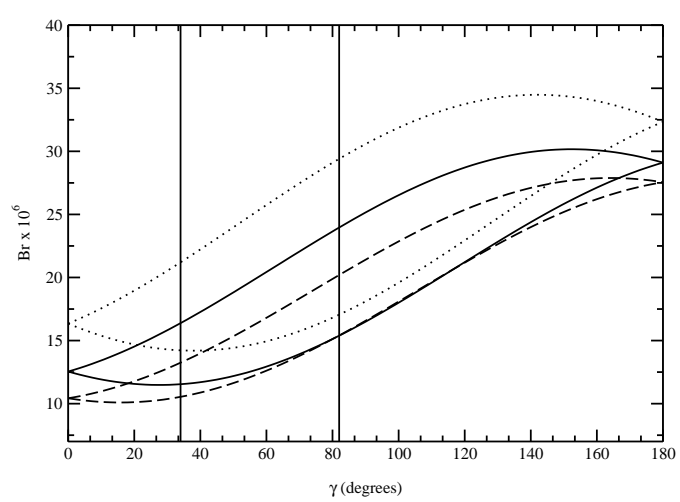

Figure 4. The impact of IC in the B meson on the $B \rightarrow K^{ \pm} \pi^{\mp}$ branching ratios as a function of $\gamma$. The upper curve for each type of line corresponds to $B^{0} \rightarrow K^{+} \pi^{-}$decay, whereas the lower curve for each type of line corresponds to $\bar{B}^{0} \rightarrow K^{-} \pi^{+}$decay. The solid line depicts the results of Ref. [34]. The dashed line is the result once the IC contribution, is subtracted, and the dotted line is the result once the IC contribution is added. The vertical solid lines enclose the $\geq 5 \%$ C.L. fits to $\gamma$ in the SM, $34^{\circ} \geq \gamma \geq 82^{\circ}$, of Ref. 35.].

the U.S. Department of Energy for support under contracts DE-FG02-96ER40989 and DE-AC03$76 \mathrm{SF} 00515$.

\section{REFERENCES}

1. S. J. Brodsky and S. Gardner, Phys. Rev. D 65, 054016 (2002).

2. P. A. M. Dirac, Rev. Mod. Phys. 21, 392 (1949).

3. S. J. Brodsky and G. P. Lepage, in Perturbative Quantum Chromodynamics, edited by A. H. Mueller (World Scientific, Singapore, 1989) and references therein.

4. S. J. Brodsky, P. Hoyer, C. Peterson, and N. Sakai, Phys. Lett. B 93, 451 (1980); S. J. Brodsky, C. Peterson, and N. Sakai, Phys. Rev. D 23, 2745 (1981).

5. A. I. Signal and A. W. Thomas, Phys. Lett. B 191, 205 (1987); M. Burkardt and B. Warr, Phys. Rev. D 45, 958 (1992); S. J. Brodsky 
and B. Q. Ma, Phys. Lett. B 381, 317 (1996); W. Melnitchouk and M. Malheiro, Phys. Rev. C 55, 431 (1997).

6. G. P. Zeller et al. [NuTeV Collaboration], Phys. Rev. D 65, 111103 (2002).

7. M. Franz, M. V. Polyakov, and K. Goeke, Phys. Rev. D 62, 074024 (2000).

8. B. W. Harris, J. Smith, and R. Vogt, Nucl. Phys. B 461, 181 (1996); F. M. Steffens, W. Melnitchouk, and A. W. Thomas, Eur. Phys. J. C 11, 673 (1999); J. Amundson, C. Schmidt, W. Tung, and X. Wang, JHEP 0010, 031 (2000).

9. S. J. Brodsky and M. Karliner, Phys. Rev. Lett. 78, 4682 (1997).

10. S. J. Brodsky, P. Hoyer, A. H. Mueller, and W. Tang, Nucl. Phys. B 369, 519 (1992); R. Vogt, S. J. Brodsky, and P. Hoyer, Nucl. Phys. B 383, 643 (1992); R. Vogt and S. J. Brodsky, Nucl. Phys. B 438, 261 (1995); Phys. Lett. B 349, 569 (1995); M. Vanttinen, P. Hoyer, S. J. Brodsky, and W. K. Tang, Phys. Rev. D 51, 3332 (1995).

11. M. Gremm, A. Kapustin, Z. Ligeti and M. B. Wise, Phys. Rev. Lett. 77, 20 (1996); C. W. Bauer and M. Trott, arXiv:hep$\mathrm{ph} / 0205039$.

12. C. V. Chang and W. Hou, Phys. Rev. D 64, 071501 (2001).

13. R. Balest et al. [CLEO Collaboration], Phys. Rev. D 52, 2661 (1995).

14. S. J. Brodsky and F. S. Navarra, Phys. Lett. B 411, 152 (1997).

15. F. Gabbiani, J.-w. Qiu, and G. Valencia, arXiv:hep-ph/0201159.

16. K. Hagiwara et al. [Particle Data Group Collaboration], Phys. Rev. D 66, 010001 (2002).

17. E. Franco, V. Lubicz, F. Mescia, and C. Tarantino, Nucl. Phys. B 633, 212 (2002) and references therein.

18. D. Waller, this conference.

19. I. Dunietz, J. Incandela, F. D. Snider, and H. Yamamoto, Eur. Phys. J. C 1, 211 (1998).

20. I. Halperin and A. Zhitnitsky, Phys. Rev. D 56, 7247 (1997); Phys. Rev. Lett. 80, 438 (1998).

21. W.-S. Hou and B. Tseng, Phys. Rev. Lett. 80, 434 (1998); A. L. Kagan and A. A. Petrov,
arXiv:hep-ph/9707354.

22. F. Yuan and K. Chao, Phys. Rev. D 56, 2495 (1997); A. A. Petrov, Phys. Rev. D 58, 054004 (1998).

23. A. J. Buras and L. Silvestrini, Nucl. Phys. B 569, 3 (2000).

24. M. Ciuchini, E. Franco, G. Martinelli, and L. Silvestrini, Nucl. Phys. B 501, 271 (1997); M. Ciuchini, R. Contino, E. Franco, G. Martinelli, and L. Silvestrini, Nucl. Phys. B 512, 3 (1998); Erratum-ibid.B531:656660,1998; M. Ciuchini, E. Franco, G. Martinelli, M. Pierini, and L. Silvestrini, Phys. Lett. B 515, 33 (2001).

25. C. Isola, M. Ladisa, G. Nardulli, T. N. Pham, and P. Santorelli, Phys. Rev. D 64, 014029 (2001) and references therein.

26. G. P. Lepage and S. J. Brodsky, Phys. Lett. B 87, 359 (1979); G. P. Lepage and S. J. Brodsky, Phys. Rev. Lett. 43, 545 (1979) [Erratum-ibid. 43, 1625 (1979)]; G. R. Farrar and D. R. Jackson, Phys. Rev. Lett. 43, 246 (1979).

27. G. P. Lepage and S. J. Brodsky, Phys. Rev. D 22, 2157 (1980).

28. A. Szczepaniak, E. M. Henley, and S. J. Brodsky, Phys. Lett. B 243, 287 (1990).

29. G. Burdman and J. F. Donoghue, Phys. Lett. B 270, 55 (1991).

30. J. Botts and G. Sterman, Nucl. Phys. B 325, 62 (1989); H. Li and G. Sterman, Nucl. Phys. B 381, 129 (1992).

31. H. N. Li and H. Yu, Phys. Rev. Lett. 74, 4388 (1995); Phys. Lett. B 353, 301 (1995); Phys. Rev. D 53, 2480 (1996).

32. M. Beneke, G. Buchalla, M. Neubert, and C. T. Sachrajda, Phys. Rev. Lett. 83, 1914 (1999); Nucl. Phys. B 591, 313 (2000); Nucl. Phys. B 606, 245 (2001).

33. S. Descotes-Genon and C. T. Sachrajda, Nucl. Phys. B 625, 239 (2002).

34. Y. Y. Keum, H. N. Li, and A. I. Sanda, Phys. Rev. D 63, 054008 (2001).

35. A. Hocker, H. Lacker, S. Laplace and F. Le Diberder, Eur. Phys. J. C 21, 225 (2001).

36. Y. Y. Keum and A. I. Sanda, arXiv:hep$\mathrm{ph} / 0209014$.

37. B. Aubert et al. [BABAR Collaboration], 
arXiv:hep-ex/0207055; B. C. Casey [Belle Collaboration], arXiv:hep-ex/0207090.

38. M. Gronau and J. L. Rosner, arXiv:hep$\mathrm{ph} / 0205323$ and references therein. 